\title{
Mining expression and prognosis of FOLR1 in ovarian cancer by using Oncomine and Kaplan- Meier plotter
}

https://doi.org/10.1515/pteridines-2019-0020

received March 20, 2019; accepted August 12, 2019.

\begin{abstract}
Objective: To further explore folate receptor 1 (FOLR1) gene expression in ovarian cancer and its association with patients' prognosis by deep mining the Oncomine and Kaplan-Meier plotter databases.
\end{abstract}

Methods: FOLR1 mRNA expression data of ovarian cancer were retrieved from the Oncomine database and further analyzed by comparing tumor to healthy tissue. The prognostic value of FOLR1 in ovarian cancer was analyzed by Kaplan-Meier Plotter, an online survival analysis database.

Results A total of 439 studies were included in the Oncomine database in multiple types of cancers. Of the 439 studies, there were 54 with statistical differences for the expression of FOLR1, 19 with increased expression of FOLR1 and 35 with decreased expression comparing ovarian cancer to normal ovary tissue. After searching the Oncomine database, six datasets were discovered comparing the mRNA expression in ovarian tumor to healthy tissue. FOLR1 mRNA expression in ovarian tumor was significantly higher than that of normal ovarian tissue (all $\mathrm{p}<0.05$ ). The Kaplan-Meier Plotter database analyzed the correlation between FOLR1 expression and ovarian cancer patient's prognosis. A significant difference of progression-free survival between FOLR1 high and low expressing groups was found in ovarian cancer patients (HR=1.14, 95\%CI: 1.00-1.29, $\mathrm{p}=0.043)$. However, the overall survival was not statistically different between high and

\footnotetext{
*Corresponding author: Ruijin Wu, Department of Gynecology, Women's Hospital School of Medicine Zhejiang University 310006, E-mail:wurj@zju.edu.cn

Qingyuan Su, Department of Gynecology, Women's Hospital School of Medicine Zhejiang University 310006; Department of Gynecology, Traditional Chinese Medicine of Anji County, Zhejiang Province, 313300 Qingyuan Lv, Department of Gynecology, Lishui People's Hospital, Zhejiang Province 323000
}

low FOLR1 expressing patients ( $\mathrm{HR}=0.95,95 \% \mathrm{CI}$ : 0.84$1.09, \mathrm{p}=0.48)$.

Conclusion FOLR1 mRNA was found to be highly expressed in ovarian tumor compared to normal ovarian tissue. Elevated FOLR1 mRNA expression was associated with the poor progression-free survival.

Keywords: Ovarian cancer; FOLR1; Oncomine, Data mining; Prognosis.

\section{Introduction}

Folate receptor 1 (FOLR1), also known as folate binding protein, is a glycosylphosphoinositol anchored membrane protein, which can mediate extracellular 5-methyltetrahydrofolate into cells and plays an important role in cell division, proliferation and tissue growth $[1,2]$. In normal tissue the gene expression level of FOLR1 is low, however, in many cancers such as lung [3, 4], breast [5] and colorectal [6] the FOLR1 expression level is elevated. In ovarian cancer, the FOLR1 gene is over expressed in tumor compared to normal ovarian tissues [7]. However, most previous studies used small sample sizes with limited statistical power and weak clinical evidence. In our study, microarray FOLR1 mRNA expression data from the Oncomine database was used to determine prognosis.

The Oncomine database (https://www.oncomine. org/) is the largest oncogene chip databases with an integrated data-mining platform aimed at mining cancer gene information $[8,9]$. At present, 715 gene expression datasets and 86,733 samples of cancerous and normal tissues have been made available. Oncomine can provide differentially expressed genes between tumor and normal tissues for many cancer types and subtypes which can be analyzed based on clinical and pathological data, differentially expressed sorting and co-expression analyses, location of differentially expressed genes, identification of target genes. These analyses can then be 
used to determine research directions, not only to save time but also reducing the cost of scientific research.

\section{Material and methods}

\section{Data extraction from Oncomine database and analysis}

The Oncomine database is a web-based gene database and an integrated data mining platform, in which data can be screened and mined in accordance with requirements. This platform aimed to stimulate discoveries from genomewide expression analyses and compare the transcriptome data in most major cancer types. In our present work, folate receptor 1 (FOLR1) gene expression levels in ovarian cancer were compared to normal ovarian tissue and extracted and analyzed by the Oncomine database platform. The data search was as follows: (1) cancer type: ovarian cancer; (2) Gene: FOLR1; (3) Data type: mRNA; (4) Analysis type: Cancer vs normal; (5) Cut-off value: $\mathrm{p}<1 \mathrm{E}-4$, fold change $>2$; gene rank=top $10 \%$.

\section{Survival analysis by Kaplan-Meier Plotter}

The prognosis, in terms of overall survival (OS) and progression-free survival (PFS), was determined using tumor FOLR1 mRNA expression levels for ovarian cancer patients and analyzed by the Kaplan-Meier plotter (www. kmplot.com). This database provided online survival analysis of lung, ovarian, gastric and breast cancer patients. In order to evaluate the prognostic significance of FOLR1 expression on ovarian patient survival, the patients were divided into two cohorts according to median FOLR1 mRNA expression (high versus low expression). The OS and PFS of the ovarian cancer patients were compared between FOLR1 high and low expressing groups.

\section{Statistical analysis}

Data were analyzed by SPSS17.0 (http://www-01.ibm. com/software/analytics/spss/) and expressed by $\bar{x} \pm s$. The between group comparison of the FOLR1 expression was conducted using a student t-test, and the counted data was expressed by rate. The between group comparison was made by $\chi^{2}$-test or Fisher's exact test. A proportional hazard ratio was used for survival analysis through
Disease Summary for FOLR1
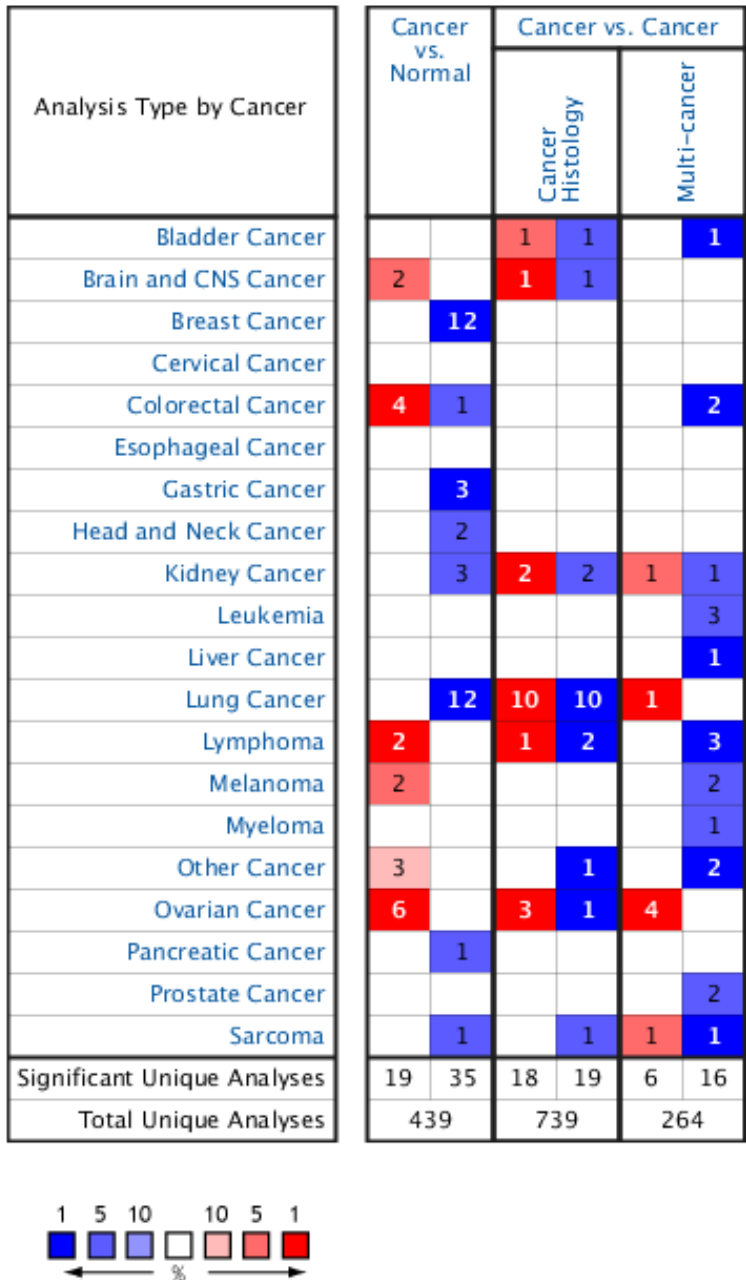

Figure 1: FOLR1 expression in multiple cancers from comparing tumor with healthy control tissue in the Oncomine database.

a log-rank test and survival curve. $\mathrm{P}<0.05$ was taken as a statistically significant difference.

\section{Results}

\section{FOLR1 expression in multiple cancers}

A total of 439 studies of multiple cancers types were included from the Oncomine database (Figure 1). Of the 439 studies, there were 54 with statistical differences for the expression of FOLR1, 19 with increased expression of FOLR1 and 35 with decreased expression when comparing ovarian tumors to normal ovarian tissues. 
Table 1: Characteristics of the included six studies.

\begin{tabular}{|c|c|c|c|c|c|}
\hline Study & Sample & size (n) & Pathology type & Microarray & Year \\
\hline & Cancer & Control & & & \\
\hline Bonome Ovarian & 185 & 10 & Serous Adenocarcinoma & Human Genome U133A Array & 2008 \\
\hline Hendrix Ovarian & 8 & 4 & Clear cell Adenocarcinoma & Human Genome U133A Array & 2006 \\
\hline Hendrix Ovarian & 37 & 4 & Endometrioid Adenocarcinoma & Human Genome U133A Array & 2006 \\
\hline Hendrix Ovarian & 41 & 4 & Serous Adenocarcinoma & Human Genome U133A Array & 2006 \\
\hline Welsh Ovarian & 28 & 4 & Ovarian Serous Surface Papillary Carcinoma & HumanGeneFL Array & 2001 \\
\hline Yoshihara Ovarian & 43 & 10 & Ovarian Serous Adenocarcinoma & Agilent Human 1A Oligo Microarray v2 & 2009 \\
\hline
\end{tabular}

\title{
Comparison of FOLR1 Across 6 Analyses
}

\author{
Over-expression
}

\begin{tabular}{|rrr|r|r|r|r|r|r|}
\hline Median Rank & p-Value & \multicolumn{9}{|l|}{ Gene } \\
\hline 113.5 & $5.18 \mathrm{E}-11$ & FOLR1 & \multicolumn{3}{|l|}{$\mid$} & \multicolumn{3}{|l|}{} \\
\hline & & 1 & 2 & 3 & 4 & 5 & 6 \\
\hline
\end{tabular}

Legend

1. Ovarian Carcinoma vs. Normal Bonome Ovarian, Cancer Res, 2008

2. Ovarian Clear Cell Adenocarcinoma vs. Normal

Hendrix Ovarian, Cancer Res, 2006

3. Ovarian Endometrioid Adenocarcinoma vs. Normal

Hendrix Ovarian, Cancer Res, 2006
4. Ovarian Serous Adenocarcinoma vs. Normal Hendrix Ovarian, Cancer Res, 2006

5. Ovarian Serous Surface Papillary Carcinoma vs. Normal Welsh Ovarian, Proc Natl Acad Sci US A, 2001

6. Ovarian Serous Adenocarcinoma vs. Normal Yoshihara Ovarian, Cancer Sci, 2009

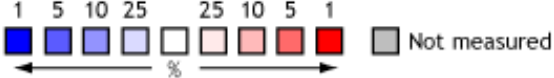

The rank for a gene is the median rank for that gene across each of the analyses.

Figure 2: Expression of FOLR1 in ovarian cancer in the studies identified by the Oncomine database. 1-6 represent the six datasets expressing FOLR1 in ovarian tumors. The darker the red, the higher the FOLR1 gene expression from the microarray.

\section{FOLR1 expression in ovarian cancer}

After searching the Oncomine database, six datasets [10-15] were discovered after comparing the mRNA expression in tumor with normal tissue (Table 1). FOLR1 was highly expressed in ovarian tumors when compared to normal ovarian tissue in all of the six series, Figure 2.

\section{FOLR1 expression by microarray analysis}

FOLR1 mRNA expression in ovarian tumor versus normal ovarian tissue by microarray analysis of the six data sets were shown in Figure 3. FOLR1 mRNA expression in ovarian tumor was significantly higher than that from normal ovary (all $\mathrm{p}<0.05$ ).

\section{FOLR1 expression and prognosis}

The correlation between FOLR1 expression and ovarian cancer patients prognosis was analyzed by the KaplanMeier Plotter database[16]. A significant difference between PFS and FOLR1 high and low expressing groups was found in ovarian cancer patients (HR=1.14, 95\%CI: 1.00-1.29, $\mathrm{p}=0.043)$. However, the OS was not statistically significantly different between high and low FOLR1 expressing patients (HR=0.95, 95\%CI: 0.84-1.09, $\mathrm{p}=0.48$ ), Figure 4. We further performed subgroup survival analysis of FOLR1 expression according to clinical stage and histology, Table 2. The prognostic significance of FOLR1 expression for subgroup analysis was demonstrated in Figure 5. The PFS was different between FOLR1 high and low expressing groups in stage 3 and 4 subgroups and 

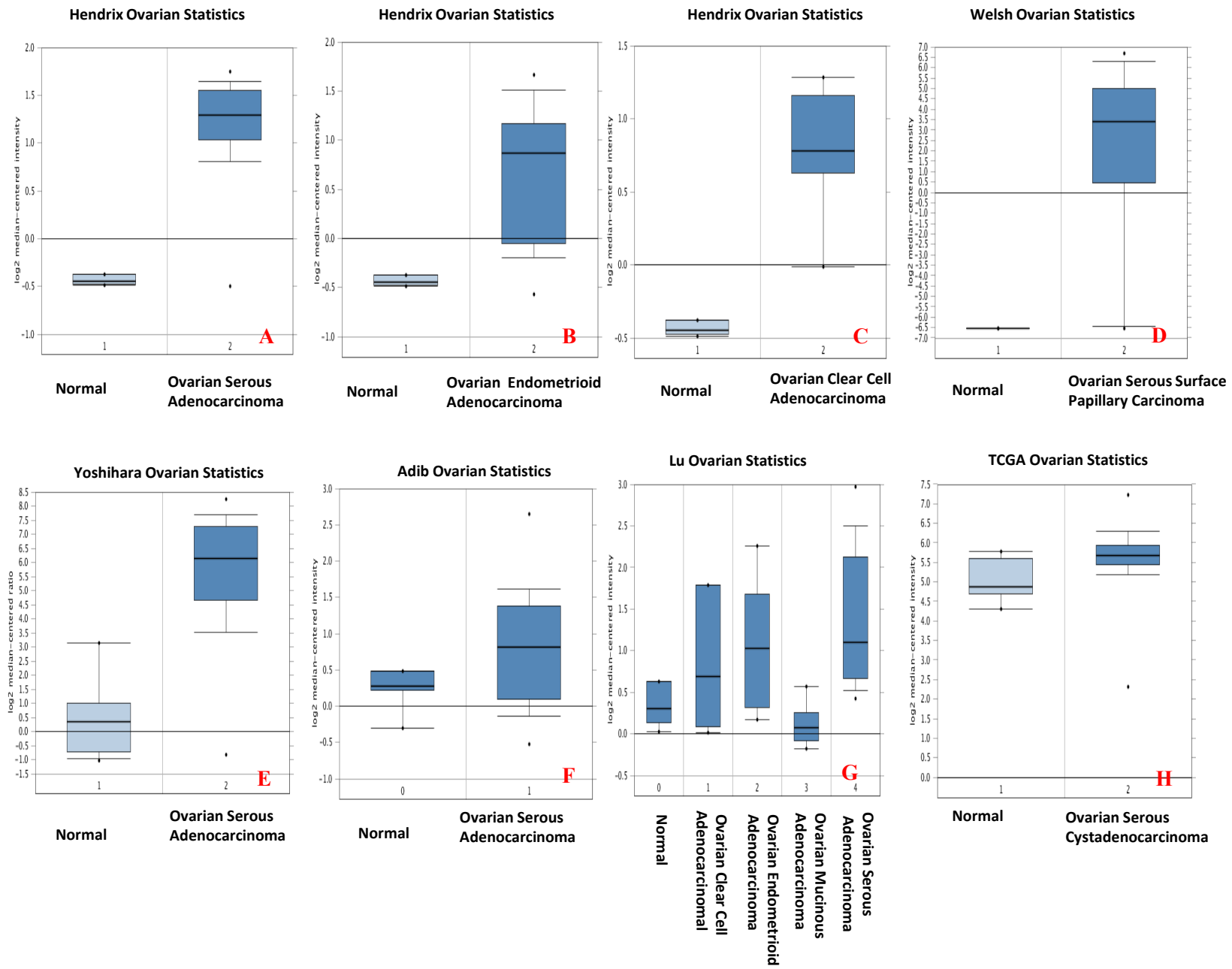

Figure 3: Expression of FOLR1 in ovarian cancer in different studies identified from the Oncomine database. (A: data form Hendrix ovarian statistics for normal tissue versus ovarian serous adenocarcinoma; B:data from Hendrix ovarian statistics for normal tissue versus ovarian endometrioid adenocarcinoma; C: data from Hendrix ovarian statistics for normal tissue versus ovarian clear cell adenocarcinoma; D: data from Welsh ovarian statistics for normal tissue versus ovarian serous surface papillary adenocarcinoma; E: data from Yoshihara ovarian statistics for normal tissue versus ovarian serous adenocarcinoma; F: data from Adlb ovarian statistics for normal tissue versus ovarian serous adenocarcinoma; G: data from Lu ovarian statistics for normal tissue versus several kinds of adenocarcinoma; H: data from TCGA databases for normal tissue versus ovarian serous cystadenocarcinoma).
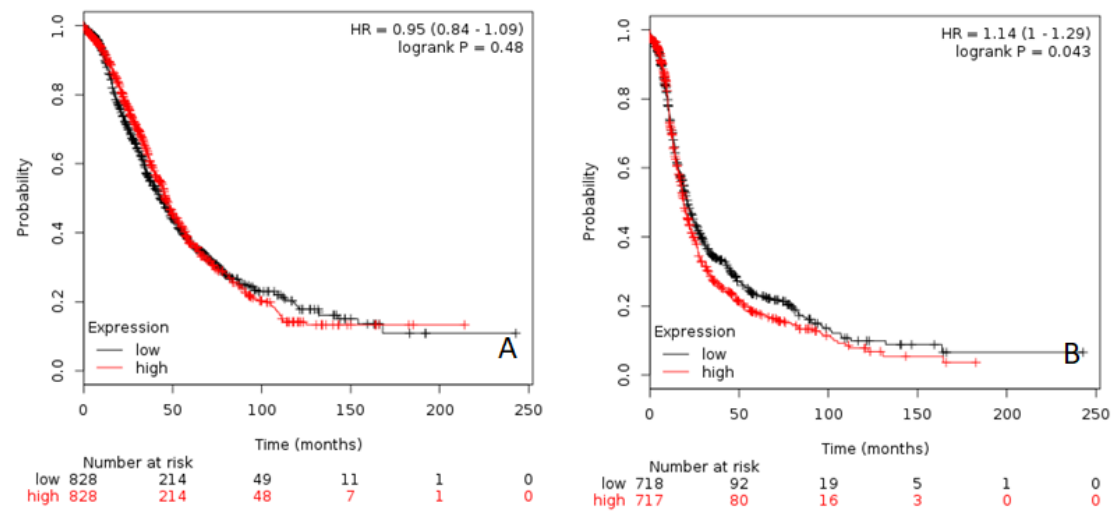

Figure 4: Overall survival (OS) and progression-free survival (PFS) curve of ovarian cancer (A: OS of ovarian cancer; B: PFS of ovarian cancer) 
Table 2: Subgroup analysis of FOLR1 gene expression and patient prognosis according to stage and history. (NA = not applicable, HR = Hazard ratio, $\mathrm{OS}=$ overall survival, $\mathrm{Cl}=$ confidence interval).

\begin{tabular}{|c|c|c|c|c|c|c|c|c|}
\hline \multirow[t]{2}{*}{ Group } & \multicolumn{2}{|c|}{ Median PFS } & \multirow[t]{2}{*}{$\mathrm{HR}(95 \% \mathrm{Cl})$} & \multirow[t]{2}{*}{ p-value } & \multicolumn{2}{|c|}{ Median OS } & \multirow[t]{2}{*}{$\mathrm{HR}(95 \% \mathrm{Cl})$} & \multirow[t]{2}{*}{ p-value } \\
\hline & High & Low & & & High & Low & & \\
\hline \multicolumn{9}{|l|}{ Stage } \\
\hline 1 & NA & NA & $1.40(0.48-4.06)$ & 0.54 & 63.90 & 81.23 & $1.06(0.34-3.28)$ & 0.92 \\
\hline 2 & 35.19 & 39.00 & $1.52(0.75-3.09)$ & 0.25 & 71.61 & 44.30 & $0.38(0.13-1.14)$ & 0.073 \\
\hline 3 & 18.83 & 15.00 & $0.81(0.70-0.95)$ & 0.0082 & 48.37 & 40.13 & $0.83(0.71-0.99)$ & 0.033 \\
\hline 4 & 13.00 & 20.07 & $1.86(1.23-2.81)$ & 0.0029 & 27.30 & 38.40 & $1.48(1.02-2.14)$ & 0.037 \\
\hline \multicolumn{9}{|l|}{ Histology } \\
\hline Endometrioid & 9.00 & 34.00 & $4.83(1.84-12.66)$ & 0.00046 & NA & NA & $0.44(0.07-2.64)$ & 0.36 \\
\hline Serous & 17.43 & 18.40 & $1.12(0.95-1.33)$ & 0.17 & 48.37 & 40.10 & $0.81(0.70-0.95)$ & 0.0097 \\
\hline
\end{tabular}
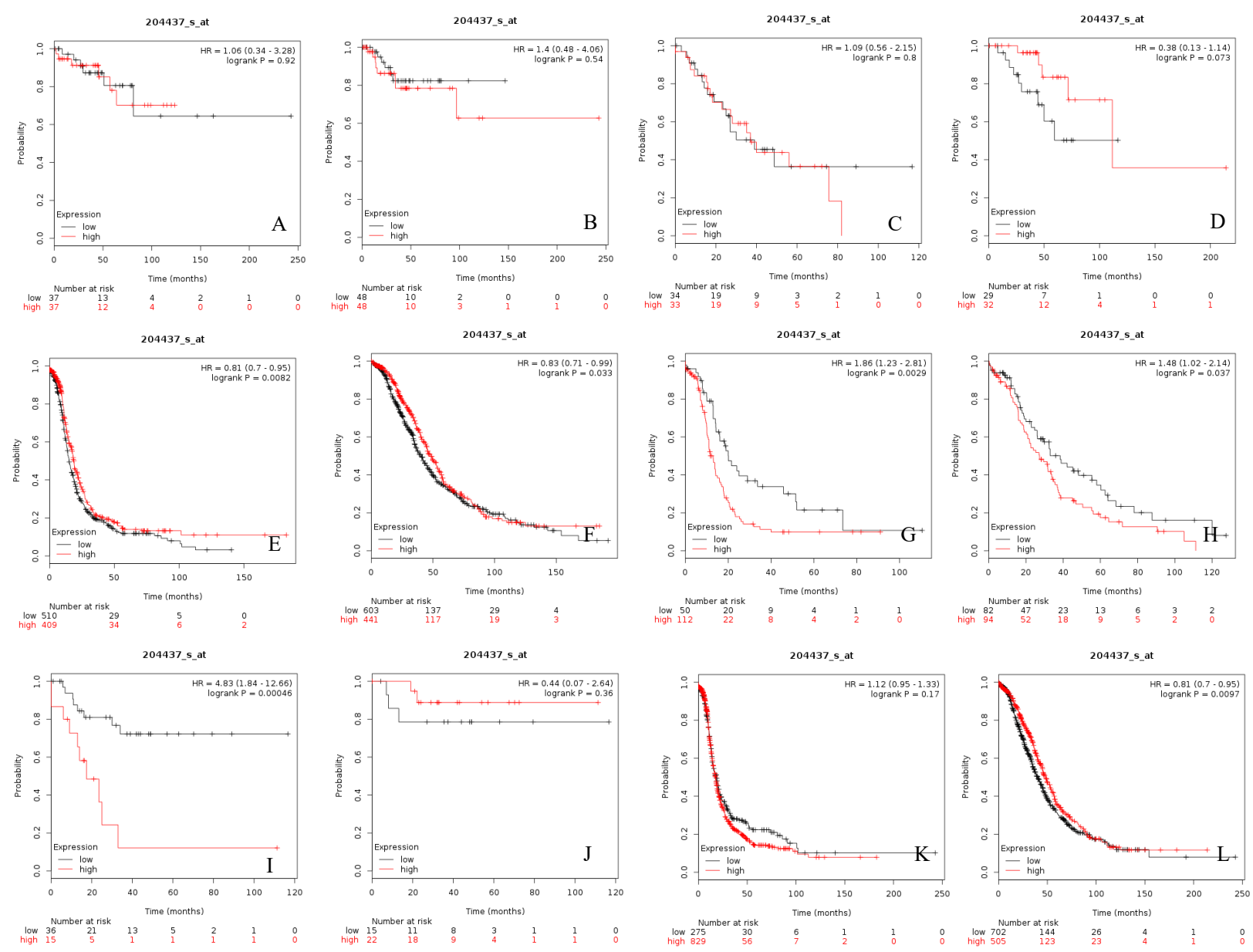

Figure 5: Subgroup survival analysis for FOLR1 gene expression survival curves according to clinical stage and histology (A: OS of stage 1 ovarian cancer; B: PFS of stage 1 ovarian cancer; C: PFS of stage 2 ovarian cancer; D: OS of stage 2 ovarian cancer; E: PFS of stage 3 ovarian cancer; F: OS of stage 3 ovarian cancer; G: PFS of stage 4 ovarian cancer; H: OS of stage 4 ovarian cancer; I: DFS of endometrioid ovarian cancer; J: OS of endometrioid ovarian cancer; K:DFS of serous ovarian cancer; L: OS of serous ovarian cancer). 
endometrioid histology subgroup $(\mathrm{p}<0.05)$. For OS, there was a statistical difference in stage 3 and 4 subgroups and serous histology subgroup $(\mathrm{p}<0.05)$.

\section{Discussion}

Ovarian tumors are one of the most common carcinomas in female genital organs [17]. The incidence of ovarian cancer ranks third, after cervical and uterine body cancers $[18,19]$. However, the mortality rate of epithelial ovarian cancer is the highest among all gynecological carcinomas [20]. The cancer epidemiological study from the United States in 2019, showed that there were 22,530 new cases of ovarian cancer and 19,380 deaths, and only $\sim 40 \%$ of ovarian cancer patients were suitable for curative resection [17]. Epithelial cancer is the most common malignant ovarian tumors, followed by malignant germ cell tumors [21]. In patients with ovarian cancer, only 30\% of the tumors were discovered to be confined to the ovary $[22,23]$, the majority had spread to the uterus, bilateral appendages, omentum and pelvic organs [23, 24].

The prognosis of patients with ovarian cancer is poor when diagnosed in advanced stages, and much more is needed to be done to improve early diagnostic methods. There are many factors which can affect the prognosis of ovarian cancer patients such as clinical stage, pathological classification, post-operative treatment, and surgical methods $[25,26]$. Studies have also shown that the expression of a variety of genes negatively correlates to patient prognoses, such as CA-125 $[27,28]$ and FOLR1 [29].

FOLR1 is a member of the human folate-binding protein family (three isomers of folate-binding protein have been identified: FOLR1, FOLR2 and FOLR3). The gene is located on chromosome 11q13.3-13.5. Its protein is exposed to the outside of the cell and anchored by GPI on the cell membrane. FOLR1 transports extracellular 5-methyltetrahydrofolate into the cell and participates in DNA replication and replication as a coenzyme component. The expression level of FOLR1 is closely related to tumor progression and cell proliferation. Several studies have evaluated FOLR1 expression and ovarian cancer patient prognosis, but the results were inconclusive [29-31]. Kimberly et al. [32] examined FOLR1 protein expression in 186 primary ovarian cancer patients and 27 cases of recurrence disease by immunohistochemical assay. The authors found that the expression of FOLR1 did not correlate with the OS of the ovarian cancer patients. However, Fu et al. [33] evaluated the correlation between
FOLR1 mRNA expression and prognosis in patients with serous ovarian carcinoma by RT-PCR. They found high expressing FOLR1 was correlated with poor prognosis, which was in accordance with our results.

In our present work, six comparisons of tumor versus normal tissue from ovarian cancer patients were included here. FOLR1 gene expression in ovarian cancer was analyzed and compared with normal ovarian tissue. Of the six data sets, three were from three studies which compared three different ovarian cancer subtypes. All six data sets confirmed FOLR1 gene expression levels from tumor were higher when compared to healthy control tissue. This may indicate that FOLR1 plays an important role in ovarian cancer development. We also found that elevated FOLR1 mRNA expression was associated with poor PFS. The correlation between elevated FOLR1 mRNA expression and poor prognosis indicated that FOLR1 mRNA could be used as a molecular biomarker for the prediction of ovarian cancer patient prognosis.

\section{Conclusion}

The deep mining of FOLR1 gene expression confirmed that the gene is highly expressed in ovarian tumors, and negatively correlates with ovarian cancer patient prognosis.

Conflict of interest: Authors state no conflict of interest

\section{Founding: None}

\section{References}

1. Assaraf YG, Leamon CP, Reddy JA. The folate receptor as a rational therapeutic target for personalized cancer treatment. Drug Resist Updat 2014;17:89-95.

2. Frigerio B, Bizzoni C, Jansen G, Leamon CP, Peters GJ, Low PS, Matherly LH, et al.. Folate receptors and transporters: biological role and diagnostic/therapeutic targets in cancer and other diseases. J Exp Clin Cancer Res 2019;38:125.3.

3. Christoph DC, Asuncion BR, Hassan B, Tran C, Maltzman JD, O'Shannessy DJ, et al. Significance of folate receptor alpha and thymidylate synthase protein expression in patients with nonsmall-cell lung cancer treated with pemetrexed. J Thorac Oncol 2013;8:19-30.

4 Kato T, Jin CS, Ujiie H, Lee D, Fujino K, Wada $\mathrm{H}$, et al. Nanoparticle targeted folate receptor 1-enhanced photodynamic therapy for lung cancer. Lung Cancer 2017;113:59-68. 
5. O'Shannessy DJ, Somers EB, Maltzman J, Smale R, Fu YS. Folate receptor alpha (FRA) expression in breast cancer: identification of a new molecular subtype and association with triple negative disease. Springerplus 2012;1:22.

6. D’Angelica M, Ammori J, Gonen M, Klimstra DS, Low PS, Murphy $L$, et al. Folate receptor- $\alpha$ expression in resectable hepatic colorectal cancer metastases: patterns and significance. Mod Pathol 2011;24:1221-8.

7. Despierre E, Lambrechts S, Leunen K, Berteloot P, Neven P, Amant $F$, et al. Folate receptor alpha (FRA) expression remains unchanged in epithelial ovarian and endometrial cancer after chemotherapy. Gynecol Oncol 2013;130:192-9.

8. Rhodes DR, Kalyana-Sundaram S, Mahavisno V, Varambally $\mathrm{R}, \mathrm{Yu}$ J, Briggs BB, et al. Oncomine 3.0: genes, pathways, and networks in a collection of 18,000 cancer gene expression profiles. Neoplasia 2007;9:166-80.

9. Rhodes DR, Yu J, Shanker K, Deshpande N, Varambally R, Ghosh D, et al. ONCOMINE: a cancer microarray database and integrated data-mining platform. Neoplasia 2004;6:1-6.

10. Hendrix ND, Wu R, Kuick R, Schwartz DR, Fearon ER, Cho KR. Fibroblast growth factor 9 has oncogenic activity and is a downstream target of Wnt signaling in ovarian endometrioid adenocarcinomas. Cancer Res 2006;66:1354-62.

11. Welsh JB, Zarrinkar PP, Sapinoso LM, Kern SG, Behling CA, Monk BJ, et al. Analysis of gene expression profiles in normal and neoplastic ovarian tissue samples identifies candidate molecular markers of epithelial ovarian cancer. Proc Natl Acad Sci U S A 2001;98:1176-81.

12. Yoshihara K, Tajima A, Komata D, Yamamoto T, Kodama S, Fujiwara $\mathrm{H}$, et al. Gene expression profiling of advanced-stage serous ovarian cancers distinguishes novel subclasses and implicates ZEB2 in tumor progression and prognosis. Cancer Sci 2009;100:1421-8.

13. Bonome T, Levine DA, Shih J, Randonovich M, Pise-Masison CA, Bogomolniy $\mathrm{F}$, et al. A gene signature predicting for survival in suboptimally debulked patients with ovarian cancer. Cancer Res 2008;68:5478-86.

14. Adib TR, Henderson S, Perrett C, Hewitt D, Bourmpoulia D, Ledermann J, et al. Predicting biomarkers for ovarian cancer using gene-expression microarrays. Br J Cancer 2004;90:68692.

15. Lu KH, Patterson AP, Wang L, Marquez RT, Atkinson EN, Baggerly KA, et al. Selection of potential markers for epithelial ovarian cancer with gene expression arrays and recursive descent partition analysis. Clin Cancer Res 2004;10:3291-300.

16. Gyorffy B, Lánczky A, Szállási Z. Implementing an online tool for genome-wide validation of survival-associated biomarkers in ovarian-cancer using microarray data from 1287 patients. Endocr Relat Cancer 2012;19:197-208.

17. Siegel RL, Miller KD, Jemal A. Cancer statistics, 2019. CA Cancer J Clin 2019;69:7-34.

18. Siegel RL, Miller KD, Jemal A. Cancer Statistics, 2017. CA Cancer I Clin 2017;67:7-30.

19. Siegel RL, Miller KD, Jemal A. Cancer statistics, 2016. CA Cancer J Clin 2016;66:7-30.

20. Torre LA, Trabert B, DeSantis CE, Miller KD, Samimi G, Runowicz CD, et al. Ovarian cancer statistics, 2018. CA Cancer J Clin 2018;68:284-296

21. Webb PM, Jordan SJ. Epidemiology of epithelial ovarian cancer. Best Pract Res Clin Obstet Gynaecol 2017;41:3-14.
22. Reid BM, Permuth JB, Sellers TA. Epidemiology of ovarian cancer: a review. Cancer Biol Med 2017;14:9-32.

23. Kanninen TT, Nasioudis D, Sisti G, Holcomb K, Di TM, Khalil S, et al. Epidemiology of Second Primary Tumors in Women With Ovarian Cancer. Int J Gynecol Cancer 2017;27:659-667.

24. Jessmon P, Boulanger T, Zhou W, Patwardhan P. Epidemiology and treatment patterns of epithelial ovarian cancer. Expert Rev Anticancer Ther 2017;17:427-437.

25. Zavesky L, Jancarkova N, Kohoutova M. Ovarian cancer: origin and factors involved in carcinogenesis with potential use in diagnosis, treatment and prognosis of the disease. Neoplasma 2011;58:457-68.

26. Vini L, Hyer S, Al-Saadi A, Pratt B, Harmer C. Prognosis for fertility and ovarian function after treatment with radioiodine for thyroid cancer. Postgrad Med J 2002;78:92-3.

27. Díaz-Padilla I, Razak AR, Minig L, Bernardini MQ, María DCJ. Prognostic and predictive value of CA-125 in the primary treatment of epithelial ovarian cancer: potentials and pitfalls. Clin Transl Oncol 2012;14:15-20.

28. Cohen AD, Piura B, Cohen Y, Shoenfeld Y. [CA 125 in diagnosis and prognosis of epithelial ovarian cancer]. Harefuah 1994;126:283-5, 303.

29. Kurosaki A, Hasegawa K, Kato T, Abe K, Hanaoka T, Miyara A, et al. Serum folate receptor alpha as a biomarker for ovarian cancer: Implications for diagnosis, prognosis and predicting its local tumor expression. Int J Cancer 2016;138:1994-2002.

30. Bergamini A, Ferrero S, Leone RMU, Scala C, Pella F, Vellone VG, et al. Folate receptor alpha antagonists in preclinical and early stage clinical development for the treatment of epithelial ovarian cancer. Expert Opin Investig Drugs 2016;25:1405-1412.

31. Vergote IB, Marth C, Coleman RL. Role of the folate receptor in ovarian cancer treatment: evidence, mechanism, and clinical implications. Cancer Metastasis Rev 2015;34:41-52.

32. Kalli KR, Oberg AL, Keeney GL, Christianson TJ, Low PS, Knutson KL, et al. Folate receptor alpha as a tumor target in epithelial ovarian cancer. Gynecol Oncol 2008;108:619-26.

33. Jifen F, Xiongyue P, Aili W. Association of folate receptor $\alpha$ with survival rates in serous ovarian carcinoma. China Journal of Modern Medicine 2018;28:31-36. 